PROCEEDINGS OF THE

AMERICAN MATHEMATICAL SOCIETY

Volume 134, Number 2, Pages 365-370

S 0002-9939(05)08343-7

Article electronically published on September 20, 2005

\title{
COMPACT PERTURBATIONS OF $m$-ACCRETIVE OPERATORS IN BANACH SPACES
}

\author{
CLAUDIO H. MORALES
}

(Communicated by Jonathan M. Borwein)

\begin{abstract}
This paper continues a discussion that arose twenty years ago, concerning the perturbation of an $m$-accretive operator by a compact mapping in Banach spaces. Indeed, if $A$ is $m$-accretive and $g$ is compact, then the boundary condition $t x \notin A(x) g(x)$ for $x \in \partial G \cap D(A)$ and $t<0$ implies that 0 is in the closure of the range of $A+g$. Perhaps the most interesting aspect of this result is the proof itself, which does not appeal to the classical degree theory argument used for this type of problem.
\end{abstract}

\section{INTRODUCTION}

Let $X$ be a (real) Banach space. An operator $A: D(A) \subset X \rightarrow 2^{X}$ is said to be $k$-accretive $(k \in \mathbb{R})$ if for each $u, v \in D(A)$ there exists $j \in J(u-v)$ such that

$$
\langle u-v, j\rangle \geq k\|x-y\|^{2}
$$

for all $u \in A x$ and $y \in A y$, where $J: X \rightarrow X^{*}$ is the normalized duality mapping which is defined by

$$
J(u)=\left\{j \in X^{*}:\langle u, j\rangle=\|u\|^{2},\|j\|=\|u\|\right\} .
$$

Here $\langle.,$.$\rangle denotes the generalized duality pairing. For k>0$ in the inequality (1), we say that $A$ is strongly accretive, while for $k=0, A$ is called accretive. In addition, if the range of $I+\lambda A$ is precisely $X$ for $\lambda>0$, then $A$ is said to be $m$-accretive. However, mappings of the form $I-A$ are known to be $k$-pseudo-contractive (see [10]). Indeed, $T$ is said to be $k$-pseudo-contractive if for each $x, y \in D(T)$ there exists $j \in J(x-y)$ such that

$$
\langle u-v, j\rangle \leq k\|x-y\|^{2}
$$

for all $u \in T x$ and $v \in T y$. If $k<1, T$ is called strongly pseudo-contractive, while for $k=1, T$ is called pseudo-contractive. This latter class of operators was first introduced by Browder [2], while the accretive mappings were independently introduced by Browder 1 and Kato 9 .

Our main purpose here is to study the existence of solutions for equations involving $m$-accretive operators perturbed by compact mappings under the LeraySchauder condition. For the last twenty years, this problem has been extensively studied under stronger conditions. Among the contributors we mention Kartsatos

Received by the editors February 5, 2004.

2000 Mathematics Subject Classification. Primary 47H10.

Key words and phrases. $m$-accretive operators, pseudo-contractive and compact operators.

(C)2005 American Mathematical Society Reverts to public domain 28 years from publication 
[6], 7], Hirano [4, Hirano and Kalinde [5], Morales [12, and Yang [15. In fact, Theorem 1 below represents a significant extension of known results of this nature. However, the most revealing aspect of this work is the fact of the results are obtained without even indirect recourse to degree theory arguments, which is contrary to what has been done in previous works of this nature.

\section{Preliminaries}

We begin with a result that is a special case of Proposition 1 of Morales [14.

Proposition 1. Let $X$ be a Banach space and let $K$ be a closed convex subset of $X$. Let $D$ be an open subset of $K$ with $0 \in D$. Suppose $g: \bar{D} \rightarrow K$ is a compact mapping satisfying the Leray-Schauder condition:

$$
g(x) \neq \lambda x \text { for all } x \in \partial_{K} D \text { and } \lambda>1 .
$$

If, in addition, the set $\{x \in D: g(x)=\lambda x$ for some $\lambda>1\}$ is bounded, then $g$ has a fixed point in $\bar{D}$.

Corollary 1. Let $X$ be a Banach space and let $K$ be a closed convex subset of $X$ with $0 \in K$. Suppose $g: K \rightarrow K$ is a compact mapping such that the set

$$
F=\{x \in K: g(x)=\lambda x \text { for some } \lambda>1\}
$$

is bounded. Then $g$ has a fixed point in $\bar{D}$.

\section{MAIN RESULTS}

The following proposition will be needed in the proof of our main result.

Proposition 2. Let $X$ be a Banach space, let $T: D(T) \subset X \rightarrow 2^{X}$ be strongly pseudo-contractive with $0 \in \overline{D(T)}$ and let $g: \overline{D(T)} \subset X \rightarrow X$ be compact. Then:

(i) The mapping $\lambda \mapsto x_{\lambda}$ with $\lambda x \in T\left(x_{\lambda}\right)+g\left(x_{\lambda}\right)$ for $\lambda>1$ is continuous provided that the set $E=\left\{x_{\lambda}: \lambda>1\right\}$ is bounded.

(ii) Let $G$ be a bounded and open subset of $X$ containing the origin for which $\lambda x \notin T(x)+g(x)$ for $x \in \partial G \cap D(A)$ and $\lambda>1$. Suppose $x_{\lambda}$ is defined for each $\lambda \geq \lambda_{0}>1$ and $x_{\mu} \in G$ for some $\mu \geq \lambda_{0}$. Then $x_{\lambda} \in G$ for all $\lambda$.

(iii) Suppose there exists $\lambda_{0}>1$ such that $\lambda x_{\lambda} \in T\left(x_{\lambda}\right)+g\left(x_{\lambda}\right)$ for $\lambda>\lambda_{0}$ with $\left\{g\left(x_{\lambda}\right): \lambda>\lambda_{0}\right\}$ bounded. Then $x_{\lambda} \rightarrow 0$ as $\lambda \rightarrow \infty$.

Proof. (i) Let $\lambda, \mu>1$. Since $T$ is strongly pseudo-contractive, there exists $j \in$ $J\left(x_{\lambda}-x_{\mu}\right)$ such that

$$
\left\langle\lambda x_{\lambda}-\mu x_{\mu}, j\right\rangle=\langle u-v, j\rangle+\left\langle g\left(x_{\lambda}\right)-g\left(x_{\mu}\right), j\right\rangle
$$

for some $u \in T\left(x_{\lambda}\right)$ and $v \in T\left(x_{\mu}\right)$. Hence

$$
(\lambda-k)\left\|x_{\lambda}-x_{\mu}\right\| \leq|\lambda-\mu|\left\|x_{\mu}\right\|+\left\|g\left(x_{\lambda}\right)-g\left(x_{\mu}\right)\right\| .
$$

Since $g$ is compact and $E$ is bounded, we may easily derive the continuity of the mapping $\lambda \mapsto x_{\lambda}$ as a consequence of the line above.

(ii) By part (i), the set $\left\{x_{\lambda}: \lambda \geq \lambda_{0}\right\}$ is a path with $x_{\mu} \in G$. Therefore the boundary condition forces the entire path to remain in $G$. 
(iii) For each $\epsilon>0$, there exists $x \in D(T)$ such that $\|x\|<\epsilon$. Now we choose $j \in J\left(x_{\lambda}-x\right)$ and $u \in T(x)$ to derive that $\left\langle\lambda x_{\lambda}-g\left(x_{\lambda}\right)-u, j\right\rangle \leq k\left\|x_{\lambda}-x\right\|^{2}$. This implies

$$
(\lambda-k)\left\|x_{\lambda}-x\right\| \leq\left\|g\left(x_{\lambda}\right)+u\right\|+\lambda\|x\| .
$$

Hence $x_{\lambda} \rightarrow 0$ as $\lambda \rightarrow \infty$.

Theorem 1. Let $X$ be a Banach space, let $A: D(A) \subset X \rightarrow 2^{X}$ be m-accretive and let $G$ be a bounded and open subset of $X$. Let $g: \bar{G} \cap \overline{D(T)} \rightarrow X$ be compact. Suppose there exists $w \in G \cap \overline{D(T)}$ such that

$$
t(x-w) \notin A(x)+g(x) \text { for } x \in \partial G \cap D(A) \text { and } t<0 .
$$

Then $0 \in \overline{\mathcal{R}(A+g)}$.

Proof. By replacing $A$ with $A(x+w), g(x)$ with $g(x+w), D(A)$ with $D(A)-w$ and $G$ with $G-w$, we may assume that $w=0$ in (3). Now, we first solve the following problem:

$$
0 \in A(x)+g(x)+\epsilon x \text { for } 0<\epsilon<1 .
$$

To accomplish this, let $T_{\epsilon}=(1-\epsilon) I-A$. Then $T_{\epsilon}$ is strongly pseudo-contractive on $D(A)$. Since $A$ is $m$-accretive, $\left(I-t T_{\epsilon}\right)(D(A))=X$ for $t \in(0,1]$. On the other hand, by Theorem 4.1 of Dugundji $[3$, there exists a compact extension $\tilde{g}: \overline{D(A)} \rightarrow$ $X$ of the mapping $g$ such that $\tilde{g}(\overline{D(A)}) \subset \operatorname{co}(g(\bar{G} \cap \overline{D(A)}))$. Consequently, $\tilde{g}(\overline{D(A)})$ is bounded.

Let $K=[0,1] \times X$ be a subset of the Banach space $\mathbb{R} \times X$ with norm $\|(t, u)\|_{1}=$ $|t|+\|u\|$. Since $I-t T_{\epsilon}$ is expansive for each $t \in[0,1]$, then the mapping $h_{t}: X \rightarrow$ $D(A)$ defined by $h_{t}(x)=\left(I-t T_{\epsilon}\right)^{-1}(x)$ is single-valued and satisfies

$$
\left\|h_{t}(x)-h_{t}(y)\right\| \leq \frac{1}{1-t(1-\epsilon)}\|x-y\| \leq \frac{1}{\epsilon}\|x-y\| .
$$

Define the mappings $h: K \rightarrow[0,1] \times D(A)$ and $f:[0,1] \times D(A) \rightarrow K$ by $h(t, x)=\left(1, h_{t}(x)\right)$ and $f(t, x)=(t,-\tilde{g}(x))$, respectively. Then $f \circ h$ maps $K$ into $K$. Moreover,

$$
\begin{aligned}
\|h(t, x)-h(s, y)\|_{1} & \leq\|h(t, x)-h(t, y)\|_{1}+\|h(t, y)-h(s, y)\|_{1} \\
& \leq\left\|h_{t}(x)-h_{t}(y)\right\|+\left\|h_{t}(y)-h_{s}(y)\right\| \\
& \leq \frac{1}{1-t(1-\epsilon)}[\|x-y\|+\|u\||t-s|]
\end{aligned}
$$

for some $u \in T_{\epsilon}\left(h_{s}(y)\right)$. Then $h$ is continuous and bounded on $K$. On the other hand, since $\tilde{g}$ is compact, then so is $f$, which implies that $f \circ h$ is compact. We shall now show that $r(f \circ h)$ has a fixed point for each $r \in(0,1]$. To see this, we prove the set

$$
F=\{(t, x) \in K: r f(h(t, x))=\lambda(t, x) \text { for some } \lambda>1\}
$$

is bounded. Let $(t, x) \in F$. Then $f(h(t, x))=\mu(t, x)$ with $\mu>1$. This implies

$$
f\left(1, h_{t}(x)\right)=\left(1,-\tilde{g}\left(h_{t}(x)\right)\right)=(\mu t, \mu x) .
$$


Hence $\tilde{g}\left(h_{t}(x)\right)=-\mu x$, which implies $\|x\|<\|-\mu x\|=\left\|\tilde{g}\left(h_{t}(x)\right)\right\| \leq c$ for some $c>0$. Therefore, $F$ is bounded, and by Corollary $1, r(f \circ h)$ has a fixed point. This means, for each $\lambda>1$ there exists $\left(t, x_{\lambda}\right) \in K$ so that $f\left(h\left(t, x_{\lambda}\right)\right)=\lambda\left(t, x_{\lambda}\right)$, and thus

$$
f\left(1, h_{t}\left(x_{\lambda}\right)\right)=\left(1,-\tilde{g}\left(h_{t}\left(x_{\lambda}\right)\right)\right)=\left(\lambda t, \lambda x_{\lambda}\right) .
$$

Hence $\tilde{g}\left(h_{t}\left(x_{\lambda}\right)\right)=-\lambda x_{\lambda}$, while $\lambda t=1$. If $z_{\lambda}=h_{t}\left(x_{\lambda}\right)$, we obtain $x_{\lambda} \in z_{\lambda}-t T_{\epsilon}\left(z_{\lambda}\right)$ and thus $\lambda z_{\lambda} \in T_{\epsilon}\left(z_{\lambda}\right)-\tilde{g}\left(z_{\lambda}\right)$ Consequently, by Proposition 2(iii), $z_{\lambda} \rightarrow 0$ as $\lambda \rightarrow \infty$. This means, $z_{\lambda} \in G \cap D(A)$ for $\lambda$ large enough. Since $T_{\epsilon}-g$ satisfies the Leray-Schauder condition:

$$
\lambda x \notin T_{\epsilon}(x)-g(x) \text { for } x \in \partial G \cap D(A) \text { and } \lambda>1,
$$

Proposition 2(ii) implies that $z_{\lambda} \in G \cap D(A)$ for all $\lambda>1$. Therefore $\lambda z_{\lambda} \in$ $T_{\epsilon}\left(z_{\lambda}\right)-g\left(z_{\lambda}\right)$. We now let $\lambda_{n} \rightarrow 1^{+}$. Then for each $n$ there exists $z_{n} \in G \cap D(A)$ such that

$$
\lambda_{n} z_{n} \in T_{\epsilon}\left(z_{n}\right)-g\left(z_{n}\right)=(1-\epsilon) z_{n}-A\left(z_{n}\right)-g\left(z_{n}\right) .
$$

Since $\left\{z_{n}\right\}$ is bounded, we may assume, without lost of generality, that $g\left(z_{n}\right) \rightarrow y$, for some $y \in X$. Therefore, by (2), $z_{n} \rightarrow z \in \bar{G}$, and thus $\lambda_{n} z_{n}+g\left(z_{n}\right) \rightarrow z+y$. Since $A$ is a closed operator, $z \in D(A)$ and $0 \in A(z)+g(z)+\epsilon z$. We now choose a sequence $\epsilon_{n} \rightarrow 0$ as $n \rightarrow \infty$, to conclude that $0 \in \overline{\mathcal{R}(A+g)}$.

Corollary 2. Let $X$ be a Banach space, let $A: D(A) \subset X \rightarrow 2^{X}$ be $m$-accretive and let $g: \overline{D(A)} \rightarrow X$ be compact. Suppose the set

$$
E=\{x \in D(A): t x \in A(x)+g(x) \text { for } t<0\}
$$

is bounded. Then $0 \in \overline{\mathcal{R}(A+g)}$.

Proof. Since $0 \in \overline{D(A)}$ and $\mathrm{E}$ is bounded, we may select an open ball $B$ centered at the origin so that $\bar{E} \subset B$. Then

$$
t x \notin A(x)+g(x) \text { for } x \in \partial B \cap D(A) \text { and } t<0 .
$$

Therefore, by Theorem 1, the conclusion follows.

We are now ready to weaken the assumptions of Theorem 2 of Hirano [4]. However, most significantly, our proof is basically self-contained and uses elementary arguments.

Theorem 2. Let $X$ be a Banach space, let $A: D(A) \subset X \rightarrow 2^{X}$ be $m$-accretive and let $g: \overline{D(A)} \rightarrow X$ be compact. Suppose there exists a bounded open set $G$ containing the origin such that for each $x \in \partial G \cap D(A)$ there exists $j \in J(x)$, for which

$$
\langle u+g(x), j\rangle \geq 0 \text { for all } u \in A x .
$$

Then $0 \in \overline{\mathcal{R}(A+g)}$.

Proof. In view of Theorem 1, we only need to verify that condition (3) holds for $w=0$. To see this, let $t x \in A(x)+g(x)$ for some $x \in \partial G \cap D(A)$. Then there exists $u \in A x$ such that $t x=u+g(x)$. From condition (4), we derive $t\|x\|^{2} \geq 0$ and consequently, $t \geq 0$, which completes the proof. 
Theorem 3. Let $X$ be a Banach space, and let $A: D(A) \subset X \rightarrow 2^{X}$ be $m$-accretive with $(A+I)^{-1}$ compact. Let $g: \overline{D(A)} \rightarrow X$ be continuous and bounded. Assume there exists an open and bounded set $G$ containing the origin such that

$$
t x \notin A(x)+g(x) \text { for } x \in \partial G \cap D(A) \text { and } t<0 .
$$

Then $0 \in \mathcal{R}(A+g)$.

Proof. As in the proof of Theorem 1, we first solve the problem

$$
0 \in A(x)+g(x)+\epsilon x \text { for } 0<\epsilon<1 .
$$

To see this, we restrict $g$ to $\bar{G} \cap \overline{D(A)}$ and then, using Theorem 4.1 of [3] once again, we find a continuous extension $\tilde{g}: \overline{D(A)} \rightarrow X$ such that

$$
\tilde{g}(\overline{(D(A)}) \subset \operatorname{co}(g(\bar{G} \cap \overline{D(A)})),
$$

which implies that $\tilde{g}(\overline{(D(A)})$ is bounded. Also, since $(I+A)^{-1}$ is compact, so is $\left(I-t T_{\epsilon}\right)^{-1}$, where $T_{\epsilon}=(1-\epsilon) I-A$ and $t \in(0,1]$. Therefore the proof of Theorem 1 carries over, to conclude that

$$
0 \in A(x)+g(x)+\epsilon x
$$

for some $z \in \bar{G} \cap D(A)$. Select a sequence $\epsilon_{n} \rightarrow 0^{+}$. Then there exists $z_{n} \in \bar{G} \cap D(A)$ such that $0 \in A\left(z_{n}\right)+g\left(z_{n}\right)+\epsilon_{n} z_{n}$. Hence,

$$
z_{n}\left(1-\epsilon_{n}\right)-g\left(z_{n}\right) \in(I+A)\left(z_{n}\right)
$$

and thus, $z_{n}=(I+A)^{-1}\left[z_{n}\left(1-\epsilon_{n}\right)-g\left(z_{n}\right)\right]$. Since $\left\{g\left(z_{n}\right)\right\}$ is bounded and $(I+A)^{-1}$ is compact, we may obtain a convergent subsequence of $\left\{z_{n}\right\}$, which converges to a point $z \in \bar{G} \cap \bar{D}(A)$. However, the closeness of $A$, implies that $z \in D(A)$ and consequently $0 \in(A+g)(\bar{G} \cap D(A))$.

Corollary 3. Let $X$ be a Banach space, and let $A: D(A) \subset X \rightarrow 2^{X}$ be m-accretive with $(A+I)^{-1}$ compact. Let $g: \overline{D(A)} \rightarrow X$ be continuous and bounded. Assume there exists an open and bounded set $G$ containing the origin such that for each $x \in \partial G \cap D(A)$ there exists $j \in J(x)$ satisfying $\langle u+g(x), j\rangle \geq 0$ for all $u \in A x$. Then $0 \in \mathcal{R}(A+g)$.

We should observe that Corollary 3 extends Theorem 3 of Kartsatos 8 and Theorem 2 of Hirano and Kalinde [5], who assume that the boundary condition (4) must hold outside of an open ball centered at the origin with radius $b$.

\section{REFERENCES}

[1] F. F. Browder, "Nonlinear equations of evolution and nonlinear accretive operators in Banach spaces", Bull. Amer. Math. Soc. 73 (1967), 867-874. MR0232254 (38:580)

[2] F.E. Browder, "Nonlinear mappings of non-expansive and accretive type in Banach spaces", Bull. Amer. Math. Soc. 73 (1967), 875-882. MR0232255 (38:581)

[3] J. Dugundji, "An extension of Tietze's theorem", Pacific J. Math. 1 (1951), 353-367. MR0044116 (13:373c)

[4] N. Hirano, "Some surjectivity theorems for compact perturbations of accretive operators", Nonlinear Analysis, TMA, 8 (1984), 765-774. MR0750049 (86a:47054)

[5] N. Hirano and A. K. Kalinde, "On perturbations of $m$-accretive operators in Banach spaces", Proc. Amer. Math. Soc. 124 (1996), 1183-1190. MR.1301029 (96g:47058)

[6] A. G. Kartsatos, "Surjectivity results for compact perturbations of $m$-accretive operators, $J$. Math. Anal. Appl. 78 (1980), 1-16. MR0595758 (82a:47048) 
[7] A. G. Kartsatos, "Mapping theorems involving compact perturbations and compact resolvents of nonlinear operators in Banach spaces", J. Math. Anal. Appl. 80 (1981), 130-146. MR0614247 (82e:47085)

[8] A. G. Kartsatos, "On compact perturbations and compact resolvents of nonlinear $m$-accretive operators in Banach spaces", Proc. Amer. Math. Soc. 119 (1993), 1189-1199. MR1216817 (94c:47076)

[9] T. Kato, "Nonlinear semigroups and evolution equations", J. Math. Soc. Japan 19 (1967), 508-520. MR.0226230 (37:1820)

[10] C. H. Morales, "Pseudo-contractive mappings and the Leray-Schauder boundary condition," Comment. Univ. Carolinae 20 (1979), 745-756.

[11] C. H. Morales, "Nonlinear equations involving m-accretive operators", J. Math. Anal. Appl. 97 (1983), 329-336. MR0723235 (85d:47055)

[12] C. H. Morales, "Remarks on compact perturbations of $m$-accretive operators", Nonlinear Analysis, TMA 16 (1991), 771-780. MR1097130(92e:47118)

[13] C. H. Morales, "Existence theorems for strongly accretive operators in Banach spaces", SIMAA: Taylor \& Francis 4 (2002), 361-368. MR.1938855 (2003k:47081)

[14] C. H. Morales, "Strong pseudo-contractions perturbed by compact operators", Proc. Amer. Math. Soc. 133 (2005), 3613-3618.

[15] G. H. Yang, "The generalized topological degree for perturbations of $m$-accretive operators and its applications", Nonlinear Analysis, TMA 18 (1992), 403-412. MR.1152717|(93g:47076)

Department of Mathematics, University of Alabama in Huntsville, Huntsville, Alabama 35899 